\title{
Strategies for Synchronous and Multiple Metastatic Liver Tumors Designed from Epithelial-Mesenchymal Transition Concept
}

\author{
Shinji Osada*, Hisashi Imai, Yoshiyuki Sasaki, Kazuhiro Yoshida \\ Surgical Oncology, Gifu University School of Medicine, Gifu, Japan. \\ Email: *sting@gifu-u.ac.jp
}

Received April 2 ${ }^{\text {nd }}, 2012$; revised April 30 ${ }^{\text {th }}, 2012$; accepted May $9^{\text {th }}, 2012$

\begin{abstract}
At some point in the natural course of colorectal cancer up to $50 \%$ of patients will develop metastasis to the liver and it is one of the most critical effects for patient prognosis. The incidence of synchronous liver metastasis has been detected at around $20 \%-25 \%$, but the optimal timing of surgical resection remains controversial. Neoadjuvant chemotherapy has also been found to be beneficial not only for initially unresectable but also resectable synchronous metastases. Then, traditional surgical strategies of hepatic resection in accordance with past chemotherapeutic regimens have been used decreasingly over the past several years. This review will primarily discuss treatments in association with the recent developed chemotherapeutic regimens and surgical procedure from the clinical data and the concept for epithetlial-mesenchymal transition, which has recently been studied to elucidate mechanisms of the liver metastatic process.
\end{abstract}

Keywords: Colorectal Cancer; Surgical Indication; Synchronous and Multiple Liver Metastasis; Epithelial-Mesenchymal Transition (EMT)

\section{Introduction}

In spite of the developments in molecular research, procedure of diagnosis and surgical technique over the past several decades, colorectal cancer (CRC) remains a major health problem worldwide. At some point in the natural course of CRC up to $50 \%$ of patients will develop metastasis to the liver [1]. The incidence of synchronous liver metastasis, commonly defined as liver metastasis occurring within 12 months of the primary colon cancer, has been detected at around 20\% - 25\% [2]. Expansion of multidisciplinary care with advances in surgical procedure and technique in the past decade has resulted in acceptance of simultaneous resection as the standard treatment because of its safety and efficiency [3]. However, guidelines regarding the operative indications for synchronous and multiple metastases have not yet been defined. After hepatectomy to treat metastatic liver tumor, activation of the signaling pathway from c-Met-related hepatocyte growth factor (HGF) represents an important factor in the progress of liver regeneration. Therefore, designed strategy for liver metastasis should be estimated from the concept of HGF/c-Met [4]. During key biological processes such as embryonic development, tissue remodeling, restitution, or wound repair, there is a requirement

"Corresponding author. for epithelial cells to escape from their rigid structural constraints by a well-known process termed epithelialmesenchymal transition (EMT) [5]. Substantial activetion of the HGF/c-Met pathway also leads to scattering and invasion of cancer cells through activation of the cell signalling pathway, and it may regulate EMT [6].

Neoadjuvant chemotherapy has been found to be beneficial not only for initially unresectable but also resectable synchronous metastases [7,8]. After the development of combinations of 5-fluorouracil/folinic acid with irinotecan (FOLFIRI) or oxaliplatin (FOLFOX) treatment regimens, a prospective phase II study demonstrated that the response rate was $66 \%$ [9] and the maximum resection rate was $82 \%$ [10]. Traditional surgical strategies of hepatic resection in accordance with past chemotherapeutic regimens have been used decreasingly over the past several years. Therefore, this review will primarily discuss treatments in association with the FOLFOX/FOLFIRI chemotherapeutic regimens. When arguing the timing of hepatectomy and whether it should be performed first or staged, the contents and results of recent chemotherapeutic developments should be considered [11]. In the present review, chemotherapeutic strategies involving FOLFOX or FOLFIRI and/or hepatectomy will be discussed from clinical data and EMT concept. 


\section{The Role of Surgical Procedure}

The surgical indications for resection of synchronous metastasis and the optimal timing of hepatectomy (simultaneous or staged) are still controversial and widely debated [12]. Because simultaneous major hepatectomy was associated with severe morbidity rate $(36.1 \%$ vs. $17.6 \%)$ and mortality (8.3\% vs. 1.4\%) [13], a staged operation for synchronous and multiple hepatic nodules has been recommended with a delay of at least 3 months after the primary resection. In contrast, based on the results of simultaneous resection to have similar operative times, intraoperative blood loss, and complications [11], recent studies showed simultaneous resection to enhance safety $[3,14,15]$. According to the safety concepts for such surgical procedures, simultaneous colorectal and liver resections have been evaluated as grade $\mathrm{C}$ under the recommended guidelines [16], however, no randomized trials have been published.

As another side for patient benefit, the prognosis should be considered. Pre- or postoperative FOLFOX chemotherapy versus surgery alone in patients with "resectable" liver metastases was evaluated in the final report from the European Organization for Research and Treatment of Cancer (EORTC) 40983 randomized trials [7]. According to the results, chemotherapy was found to be signifycantly better than surgery alone at inducing 3-year progression-free survival ( $42.4 \%$ vs. $33.2 \%, p=0.025)$. The study proposed establishment of a new standard whereby preoperative chemotherapy is to be performed even if the tumor is resectable $[17,18]$. Resection of both intra- and extra-hepatic metastases should be considered if all metastatic sites can be completely resected and the disease is controlled by chemotherapy [19]. A recent report showed that neoadjuvant FOLFOXRI administered for 3 - 6 months is actually safe [20], and no consensus exists concerning operative mortality and morbidity rates [21, 22]. Even in the case of repeat hepatectomy, the operation itself was reported to be safe and to offer survival benefit [23]. In cases of recurrence, $70 \%$ were observed within 12 months after the initial liver operation, with 92\% observed within 24 months [24], and for disease isolated to the liver, repeat hepatic resection led to favourable patient survival $[25,26]$. The criteria for the selection of patients for hepatic re-resection included the ability to achieve an $\mathrm{R} 0$ resection, the disease-free interval, solitary recurrences, and operative risk.

A "reverse strategy", in which preoperative chemotherapy is followed by resection of the colorectal metastases and then by resection of the colorectal primary at a second operation, has been proposed for patients with advanced synchronous colorectal cancer metastases, and in particular for patients in whom the primary tumor is located in the rectum [27]. The risk for progression of metastases while the patient is undergoing treatment for the primary tumor is a concern. A recent study showed the reverse strategy to be associated with postoperative morbidity and mortality rates of $31 \%$ and $4 \%$, respectively, and a 3-year survival rate of $79 \%$ [28]. The new reverse approach includes the risk that during the period between chemotherapy and liver resection the primary tumor might become obstructive. This rare possibility can easily be solved by performing the Hartmann procedure; studies show that this "liver first" approach is a safe procedure that brings satisfactory results [29]. In fact, among 233 patients with advanced stage IV colorectal cancer, only 26 patients (11\%) had symptoms related to the primary, which was similar to the $15 \%$ rate seen in the subset of patients with rectal primaries left in place [28]. These concepts might be critical and it is expected that a series of clinical studies will be planned.

\section{Concepts Emerging from Basic Studies}

HGF and its receptor, c-Met, are well known to relate to liver regeneration. Its over-expression or activation has also been studied in the progression of CRC [30]; therefore, the c-Met pathway is indicated to play a critical role in the carcinogenesis of CRC. A report of clinical cases demonstrated that liver metastasis was significantly higher in the group with high expression of c-Met. However, in CRC cases with liver metastasis, despite highgrade immunodetection of c-Met activity in the primary tumor, these cases changed to low-grade activity in liver metastasis sites [4]. An experimental mouse study also showed that expression of c-Met decreased from culture conditions to metastasis with time and tumor size dependency [30].

Recently, some novel concepts for cancer growth and invasion have derived from EMT, whereby a cancer cell changes its cellular phenotype from a local growing type and acquires an invasive and/or metastatic ability [31]. EMT has been well recognized at the invasive margins of cancer masses, but not in localized tumors. After migrating to sites distant from the primary tumor, mesenchymal-epithelial transition (MET) is also associated with increases in mass-building activity [32]. HGF itself is involved in the regulation of not only cell growth but also cell motility and morphology [33]. By suggesting that carcinoma cells with HGF are demonstrated in relation to EMT [34], primary CRC cells with highly expressed c-Met was detected to gain motility due to HGF/receptor activation for progression to the vessels and/or distant organs (Figure 1). Therefore, it seems that the HGF/c-Met system mediates cancer progression from local expansion to distant area metastasis via the process of EMT, and is down-regulated in mass formation at secondary sites via the process of MET. In fact, the ex- 
pression of c-Met was clearly reduced in the central area, despite expression remaining high in the satellite lesions of the same tumor (Figure 2). Even within single tumours, there was a difference in c-Met expression whereby it was increased in the growing invasive periphery but decreased in the established central regions.

The expression of c-Met was demonstrated to decrease with the increase of cell density (Figure 3).

E-cadherin is a cell-cell adhesion molecule, and its loss is consistently observed at sites of EMT during cancer metastases, indicating that its level of expression correlates with cancer progression [35]. Conversely, Vimentin, another commonly used molecular marker for EMT, is well known to increase on the process of EMT [36]. Recent our study for CRC cell line [34] showed that HGF reduced the level of E-cadherin in a time-dependent manner and increased the expression of Vimentin (Figure 4(a)). Slug expression, which is one of the most common molecular factors, was also increased by HGF (Figure 4 (b)). The expression of E-cadherin has been related to chemosensitivity [37], and loss or low expression in liver metastasis occurs more frequently in CRC related to poor patient prognosis [38]. Then, neoadjuvant chemotherapy might lead therapeutic benefit for patient prognosis due to high drug effect for satellite region of metastatic tumor.

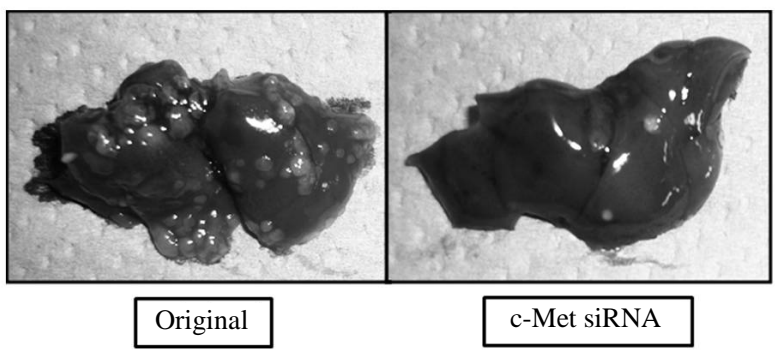

Figure 1. Effect of c-Met on liver metastasis. The protein and mRNA of c-Met were knocked down to about $30 \%$ of the control value by siRNA. On the 21 st day after injection of cancer cells in the spleen, numerous metastatic tumors were detected in the liver, but cancer cells in which c-Met expression was knocked down clearly resulted in fewer tumors.

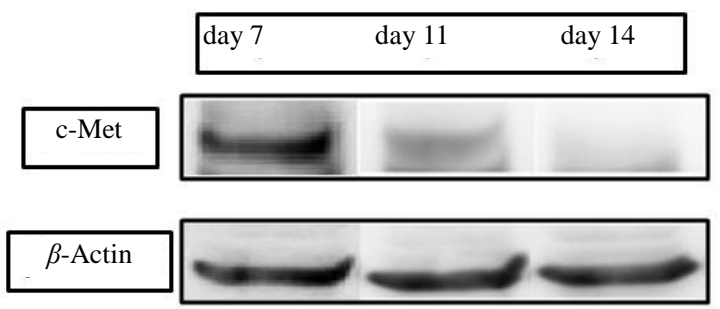

Figure 2. Expression of cellular proteins during cell proliferation. The expression of c-Met proteins decreased depending on time and increase in cell density.

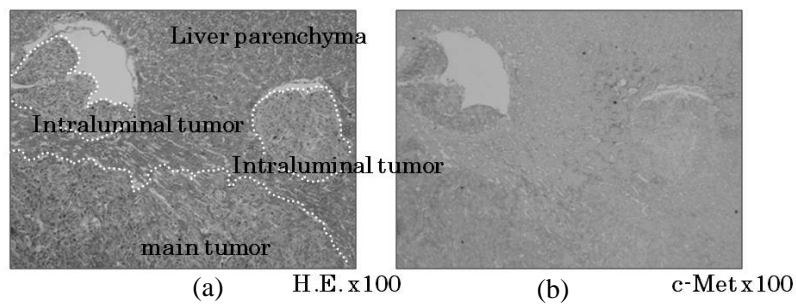

Figure 3. c-Met expression in liver metastatic sites. In the main tumor, c-Met expression was reduced in the central area but remained at high levels in satellite lesions (a). In the peripheral area of the main tumor, c-Met expression was diminished in the space completely occupied by vessels, but remained high in the space devoid of vessels (b).

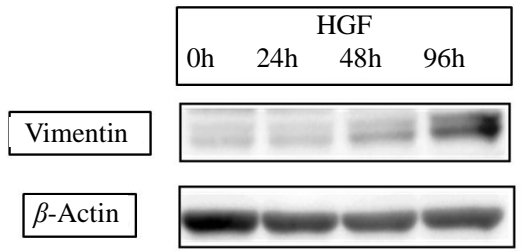

(a)

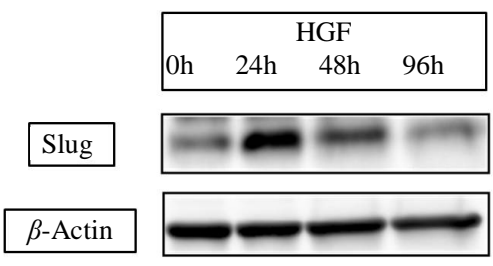

(b)

Figure 4. Expression of EMT-related proteins. (a) HGF 20 ng/ml HGF increased the expression of vimentin; (b) Slug was increased by HGF. With HGF, the peak was detected at 24 hours with a slight decrease at 48 hours.

\section{Clinical Outcomes}

Hepatectomy should be selected first if the resection can be performed safely and with curability, with no limit on the size or number of tumors. However, where curative resection is not performed for reasons such as the presence of tumors in other organs, chemotherapy should be selected first, and the timing of possible radical resection immediately planned [11].

The overall 5-year survival rate and median survival time (MST) for patients in our recent study [39] were $61.2 \%$ and $31.0 \pm 15.2$ months, respectively. Of these patients, the 3-year survival rate (55\%) and MST (28.4 \pm 15.4 months) of patients in whom synchronous liver metastasis was detected were clearly poorer than those of patients with metachronous tumors $(100 \%$ and $39.9 \pm$ 10.8 months). Among all patients studied, the 3-year survival rate and MST were significantly better $(p=0.0127)$ for patients with two or fewer tumors than for patients with three or more tumors (Figure 5(a)). Furthermore, in 
patients in whom synchronous liver tumors were detected, the 3-year survival rate and MST after staged hepatectomy were significantly better than those after simultaneous hepatectomy ( $\mathrm{p}=0.0467)$, and the MST for patients with two or fewer tumors was significantly longer than that for patients with three or more tumors (Figure 5(b)). The MST after simultaneous hepatectomy for a single tumor (29.7 \pm 8.5 months) was similar to that for multiple tumors $(23.4 \pm 15.7$ months). In contrast, after staged hepatectomy, the MSTs for patients with these factors were similar (single vs. multiple tumors, $40.7 \pm 18.3$ months vs. $30.8 \pm 11.6$ months; two or fewer vs. three or more tumors, $37.1 \pm 15.1$ months vs. $26.1 \pm 16.2$ months) [39]. Recent review demonstrated a significantly worse rate of disease-free survival after curative resection for liver metastases to be positive lymph node metastases, synchronous development timing, tumor-free interval of

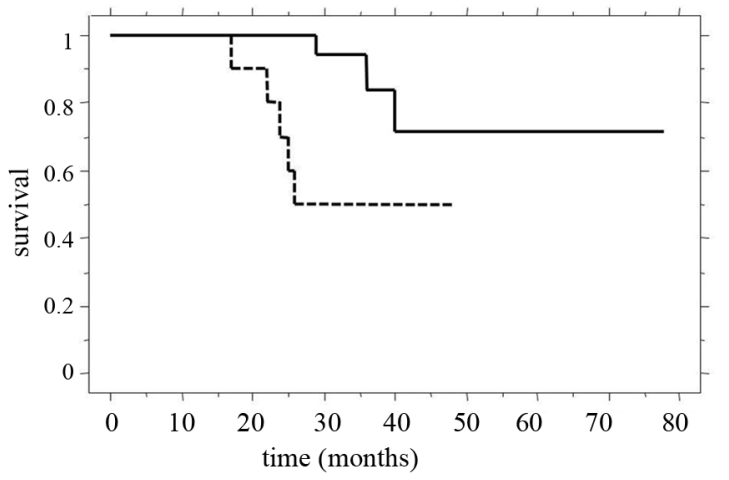

(a)

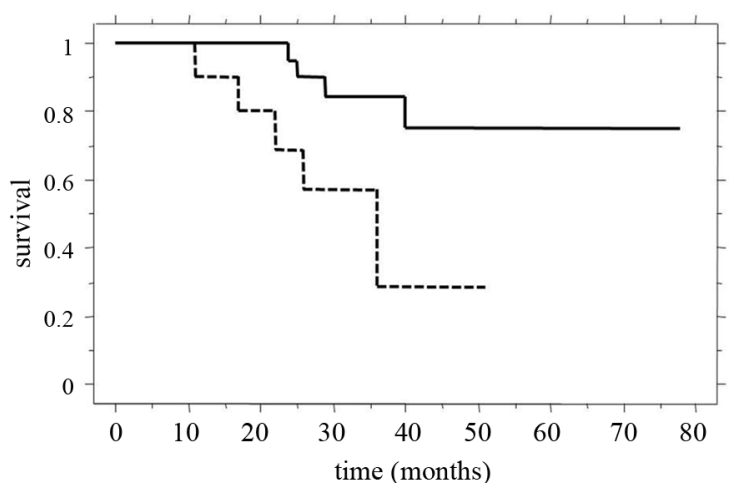

(b)

Figure 5. Clinical outcomes compared for tumor numbers. (a) The 3-year survival rate and MST of patients with two or fewer tumors (continuous line, $83 \%$ and $36.6 \pm 14.0$ months) were significantly better $(p=0.0127)$ than those of patients with three or more tumors (dotted line, $65 \%$ and 24.0 \pm 13.6 months); (b) For patients in whom synchronous liver tumors were detected, 3-year survival rate and MST after staged hepatectomy (continuous line, 82\% and $34.5 \pm$ 14.9 months) were significantly better $(p=0.0467)$ than those after simultaneous hepatectomy (dotted line, $29 \%$ and $23.9 \pm 13.6$ months). less than 12 months, presence of extrahepatic disease, and higher tumor numbers [40]. With regard to tumor number, the difference between two or fewer and three or more tumors was more critical for disease-free survival than that between one and two tumors $(p=0.001$ vs. 0.082). Another study also found three independent factors which were predictive of disease recurrence: three or more metastases at diagnosis, initial unresectability, and simultaneous colorectal operation with hepatectomy [41]. Therefore, it appears that tumor number is important for patient survival, and a cut-off point set at two tumors may be reasonable for accepting primary hepatectomy.

\section{Conclusion}

Clinical features of CRC indicate that the over-expression of c-Met is closely associated with liver metastasis. In liver metastatic lesions, although a comparative reducetion in c-Met expression correlates well with tumour growth, there is still a relatively high expression at invasive sites in the progress of EMT. Chemotherapy will lead most expected advantage for these tumor conditions. Further, clinical studies demonstrated neoadjuvant chemotherapy to improve the prognosis even for patients with synchronous liver metastases. Particularly in patients with one or two tumors, primary hepatectomy will induce a favourable outcome with a diminished likelihood of tumor in the remnant. In contrast, where there are three or more tumors, it is best to plan staged hepatictomy, even if it is technically possible to remove these tumors in one procedure. Treatment strategies for CRC patients with liver metastases should involve the consideration of appropriate combinations of chemotherapy and surgery.

\section{REFERENCES}

[1] R. Lochan, S. A. White and D. M. Manas, "Liver Resection for Colorectal Liver Metastasis,” Surgical Oncology, Vol. 16, No. 1, 2007, pp. 33-45. doi:10.1016/j.suronc.2007.04.010

[2] National Institute for Health and Clinical Excellence, "Improving Outcomes in Colorectal Cancers,” June 23, 2004.

[3] Y. Sakamoto, S. Fujita, T. Akasu, S. Nara, M. Esaki, K. Shimada, S. Yamamoto, Y. Moriya and T. Kosuge, "Is surgical Resection Justified for Stage IV Colorectal Cancer Patients Having Bilobar Hepatic Metastasis?-An Analysis of Survival of 77 Patients Undergoing Hepatectomy,” Journal of Surgical Oncology, Vol. 102, No. 7, 2010, pp. 784-788. doi:10.1002/jso.21721

[4] S. Matsui, S. Osada, H. Tomita, et al., "Clinical Significance of Aggressive Hepatectomy for Colorectal Liver Metastasis, Evaluated from the HGF/c-Met Pathway," International Journal of Oncology, Vol. 37, No. 2, 2010, pp. 289-297. 
[5] R. C. Bates and A. M. Mercurio, "The Epithelial-Mesenchymal Transition (EMT) and Colorectal Cancer Progression," Cancer Biology \& Therapy, Vol. 4, No. 4, 2005, pp. 365-370. doi:10.4161/cbt.4.4.1655

[6] H. Y. Chang, M. C. Kao, T. D. Way, C. T. Ho and E. Fu, "Diosgenin Suppresses Hepatocyte Growth Factor (HGF)Induced Epithelial-Mesenchymal Transition by DownRegulation of Mdm2 and Vimentin," Journal of Agricultural and Food Chemistry, Vol. 59, No. 10, 2011, pp. 53575363.

[7] B. Nordlinger, H. Sorbye, B. Glimelius, et al., "Perioperative Chemotherapy with FOLFOX4 and Surgery Versus Surgery Alone for Resectable Liver Metastases from Colorectal Cancer (EORTC Intergroup Trial 40983): A Randomised Controlled Trial,” The Lancet, Vol. 371, No. 9617, 2008, pp. 1007-1016. doi:10.1016/S0140-6736(08)60455-9

[8] R. Adam, G. Pascal, D. Castaing, et al., "Tumor Progression While on Chemotherapy: A Contraindication to Liver Resection for Multiple Colorectal Metastases?” Annals of Surgery, Vol. 240, No. 6, 2004, pp. 1052-1064. doi:10.1097/01.sla.0000145964.08365.01

[9] A Falcone, S Ricci, I Brunetti, et al., "Phase III Trial of Infusional Fluorouracil, Leucovorin, Oxaliplatin and Irinotecan (FOLFOXIRI) Compared with Infusional Fluorouracil, Leucovorin and Irinotecan (FOLFIRI) as FirstLine Treatment for Metastatic Colorectal Cancer,” Journal of Clinical Oncology, Vol. 25, No. 13, 2007, pp. 16701676. doi:10.1200/JCO.2006.09.0928

[10] M. Ychou, F. Viret, A. Kramar, F. Desseigne, E. Mitry, et $a l$. , "A Tritherapy with Fluorouracil/Leucovorin, Irinotecan and Oxaliplatin, FOLFILINOX, a Phase II Study in Colorectal Cancer Patients with Unresectable Liver Metastases," Cancer Chemotherapy and Pharmacology, Vol. 62, No. 2, 2008, pp. 195-201. doi:10.1007/s00280-007-0588-3

[11] S. Osada, Y. Sanada, Y. Tanaka, A. Ikawa, Y. Tokuyama, N. Okumura, K. Nonaka, T. Takahashi, K. Yamaguchi and K. Yoshida, "Strategy for Synchronous and Multiple Liver Metastasis,” Hepato-Gastroenterology, Vol. 59, No. 113, 2012, pp. 198-203.

[12] A. Brouquet, M. M. Mortenson, J. N. Vauthey, M. A. Rodriguez-Bigas, M. J. Overman, G. J. Chang, S. Kopetz, C. Garrett, S. A. Curley and E. K. Abdalla, "Surgical Strategies for Synchronous Colorectal Liver Metastases in 156 Consecutive Patients: Classic, Combined or Reverse Strategy?" Journal of the American College of Surgeons, Vol. 210, No. 6, 2010, pp. 934-941. doi:10.1016/j.jamcollsurg.2010.02.039

[13] S. K. Reddy, T. M. Pawlik, D. Zorzi, et al., "Simultaneous Resection of Colorectal Cancer and Synchronous Liver Metastases: A Multi-Institutional Analysis,” Annals of Surgical Oncology, Vol. 14, No. 12, 2007, pp. 44814491. doi:10.1245/s10434-007-9522-5

[14] S. J. Moug, D. Smith, E. Leen, C. Roxburgh and P. G. Horgan, "Evidence for a Synchronous Operative Approach in the Treatment of Colorectal Cancer with Hepatic Metastasis: A Case Matched Study," European Journal of Surgical Oncology, Vol. 36, No. 4, 2010, pp. 365-370.

\section{doi:10.1016/j.ejso.2009.11.007}

[15] J. Chen, Q. Li, C. Wang, H. Zhu, Y. Shi and G. Zhao, "Simultaneous vs. Staged Resection for Synchronous Colorectal Liver Metastases: A Meta-Analysis," International Journal of Colorectal Disease, Vol. 26, No. 2, 2011, pp. 191-199. doi:10.1007/s00384-010-1018-2

[16] J. G. Hillingso and P. Wille-Jorgensen, "Staged or Simultaneous Resection of Synchronous Liver Metastases from Colorectal Cancer: A Systematic Review," Colorectal Disease, Vol. 11, No. 1, 2009, pp. 3-10. doi:10.1111/j.1463-1318.2008.01625.x

[17] H. Sorbye, M. Mauer, T. Gruenberger, B. Glimelius, G. J. Poston, P. M. Schlag, P. Rougier, W. O. Bechstein, J. N. Primrose, E. T. Walpole, M. Finch-Jones, D. Jaeck, D. Miza, R. W. Parks, L. Collette, E. V. Cutsem, W. Scheithauer, M. P. Lutz and B. Nordlinger, "Predictive Factors for the Benefit of Perioperative FOLFOX for Resectable Liver Metastasis in Colorectal Cancer Patients (EORTC Intergroup Trial 40983),” Annals of Surgery, Vol. 255, No. 3, 2012, pp. 534-539. doi:10.1097/SLA.0b013e3182456aa2

[18] L. Spelt, B. Andersson, J. Nilsson and R. Andersson, "Prognostic Models for Outcome following Liver Resection for Colorectal Cancer Metastases: A Systematic Review," European Journal of Surgical Oncology, Vol. 38, No. 1, 2012, pp. 16-24. doi:10.1016/j.ejso.2011.10.013

[19] R. J. De Haas, D. A. Wicherts and R. Adam, "Resection of Colorectal Liver Metastases with Extrahepatic Diseases," Digestive Surgery, Vol. 25, No. 6, 2009, pp. 461466. doi:10.1159/000184737

[20] G. Msai, F. Loupakis, L. Pollina, E. Vasile, S. Cupini, S. Ricci, I. M. Brunetti, R. Ferraldeschi, G. Naso, F. Filipponi, M. Andreuccetti and A. Falcone, "Long-Term Outcome of Initially Unresectable Metastatic Colorectal Cancer Patients Treated with 5-Fluorouracil/Ieucovorin, Oxaliplatin, and Irinotecan (FOLFOXIRI) Followed by Radical Surgery of Metastases,” Annals of Surgery, Vol. 249, No. 3, 2009, pp. 420-425. doi:10.1097/SLA.0b013e31819a0486

[21] T. M. Pawlik, K. Olino, A. L. Gleisner, M. Torbenson, et al., "Preoperative Chemotherapy for Colorectal Liver Metastases: Impact on Hepatic Histology and Postoperative Outcome,” Journal of Gastrointestinal Surgery, Vol. 11, No. 7, 2007, pp. 863-868. doi:10.1007/s11605-007-0149-4

[22] D. Zorzi, A. Laurent, T. M. Pawlik, G. Y. Laumes, J. N. Vauthey and E. K. Abdalla, "Chemotherapy-Associated Hepatotoxicity and Surgery for Colorectal Liver Metastases,” British Journal of Surgery, Vol. 97, No. 3, 2007, pp. 274-286. doi:10.1002/bjs.5719

[23] R. Adam, G. Pascal, D. Azoulay, K. Tanaka, D. Castaing and H. Bismuth, "Liver Resection for Colorectal Metastases, the Third Hepatectomy," Annals of Surgery, Vol. 238, No. 6, 2003, pp. 871-884. doi:10.1097/01.sla.0000098112.04758.4e

[24] B. S. Langenhoff, P. F. M. Krabbe and T. J. M. Ruers, "Efficacy of Follow-Up after Surgical Treatment of Colorectal Liver Metastases,” European Journal of Surgical Oncology, Vol. 35, No. 2, 2009, pp. 180-186. 
[25] K. Tanaka, H. Shimada, K. Matsuo, et al., "Outcome after Simultaneous Colorectal and Hepatic Resection for Colorectal Cancer with Synchronous Metastases,” Surgery, Vol. 136, No. 3, 2004, pp. 650-659. doi:10.1016/j.surg.2004.02.012

[26] A. Antonion, R. E. Lovegrove, H. S. Tilney, et al., "Meta Analysis of Clinical Outcome after First and Second Liver Resection for Colorectal Metastases,” Surgery, Vol. 141, No. 1, 2007, pp. 9-18. doi:10.1016/j.surg.2006.07.045

[27] G. Mentha, P. E. Majno, A. Andres, et al., "Neoadjuvant Chemotherapy and Resection of Advanced Synchronous Liver Metastases before Treatment of the Colorectal Primary,” British Journal of Surgery, Vol. 93, No. 7, 2006, pp. 872-878. doi:10.1002/bjs.5346

[28] A. Brouquet, M. M. Mortenson, J. N. Vauthey, M. A. Rodriguez-Bigas, M. J. Overman, G. J. Chang, S. Kopetz, G. Christopher, S. A. Curley and E. K. Abdalla, "Surgical Strategies for Synchronous Colorectal Liver Metastases in 156 Consecutive Patients: Calssic, Combined or Reverse Strategy?” Journal of the American College of Surgeons, Vol. 210, No. 6, 2010, pp. 934-941. doi:10.1016/j.jamcollsurg.2010.02.039

[29] G. Mentha, A. D. Roth, S. Terraz, P. Giostra, A. Andres, P. Morel, L. Rubbia-Brandt and P. E. Majno, “'Liver First' Approach in the Treatment of Colorectal Cancer with Synchronous Liver Metastases,” Digestive Surgery, Vol. 25, No. 6, 2008, pp. 430-435. doi:10.1159/000184734

[30] M. Karoui, W. Koubaa, C. Delbaldo, et al., "Chemotherapy Has Also an Effect on Primary Tumor in Colon Carcinoma," Annals of Surgical Oncology, Vol. 15, No. 12, 2008, pp. 3440-3446. doi:10.1245/s10434-008-0167-9

[31] J. M. Lee, S. Dedhar, R. Kalluri and E. W. Thompson, "The Epithelial-Mesenchymal Transition: New Insights in Signaling, Development, and Disease," The Journal of Cell Biology, Vol. 172, No. 7, 2006, pp. 973-981. doi:10.1083/jcb.200601018

[32] E. Vincan and N. Barker, "The Upstream Components of the Wnt Signalling Pathway in the Dynamic EMT and MET Associated with Colorectal Cancer Progression,” Clinical and Experimental Metastasis, Vol. 25, No. 6, 2008, pp. 657-663. doi:10.1007/s10585-008-9156-4

[33] S. Osada, M. Kanematsu, H. Imai, S. Goshima and Y. Sugiyama, "Evaluation of Extracellular Signal Regulated
Kinase Expression and Its Relation to Treatment of Hepatocellular Carcinoma," Journal of the American College of Surgeons, Vol. 201, No. 3, 2005, pp. 405-411. doi:10.1016/j.jamcollsurg.2005.05.015

[34] T. Tanahashi, S. Osada, A. Yamada, J. Kato, K. Yawata, H. Imai, Y. Sasaki, S. Saito, Y. Tanaka, K. Nonaka and K. Yoshida, "Extracellular Signal-Regulated Kinase and Akt Activation Play a Critical Role in the Process of Hepatocyte Growth Factor-Induced Epithelial-Mesenchymal Transition," Cancer, 2012, in press.

[35] Y. Wu and B. P. Zhou, "New Insights of Epithelial-Mesenchymal Transition in Cancer Metastasis," Acta Biochimica et Biophysica Sinica, Vol. 40, No. 7, 2008, pp. 643650. doi:10.1111/j.1745-7270.2008.00443.x

[36] L. A. Walsh and S. Damjanovski, "IGF-1 Increases Invasive Potential of MCF 7 Breast Cancer Cells and Induces Activation of Latent TGF-Beta1 Resulting in Epithelial to Mesenchymal Transition," Cell Communication and Signaling, Vol. 9, 2011, p. 10. doi:10.1186/1478-811X-9-10

[37] T. Nakamura, Y. Kato, H. Fuji, T. Horiuchi, Y. Chiba and K. Tanaka, "E-Cadherin-Dependent Intercellular Adhesion Enhances Chemoresistance," International Journal of Molecular Medicine, Vol. 12, No. 5, 2003, pp. 693700.

[38] S. J. Yu, J. K. Yu, W. T. Ge, H. G. Hu, Y. Yuan and S. Zheng, "SPARCL1, Shp2, MSH2, E-cadherin, p53, ADCY2 and MAPK are Prognosis-Related in Colorectal Cancer,” World Journal of Gastroenterology, Vol. 17, No. 5, 2011, pp. 2028-2036. doi:10.3748/wjg.v17.i15.2028

[39] S. Osada, H. Imai, Y. Sasaki, Y. Tanaka, N. Okumura, K. Nonaka, T. Takahashi, K. Yamaguchi and K. Yoshida, "Surgical Indications for Multiple and Synchronous and Liver Metastases from Colorectal Cancer," Hepatogastroenterol, in press.

[40] U. Settmacher, Y. Dittmar, T. Knosel, U. Schone, M. Heise, K. Jandt and A. K. Altendorf-Hoffmann, "Predictors of Long-Term Survival in Patients with Colorectal Liver Metastases: A Single Center Study and Review of the Literature," International Journal of Colorectal Disease, Vol. 26, No. 8, 2011, pp. 1195-1197. doi:10.1007/s00384-011-1195-7

[41] R. J. De Haas, R. Adam, D. A. Wicherts, D. Azoulay, H. Bismuth, E. Vibert, C. Salloum, F. Perdigao, A. Benkabbou and D. Castaing, "Comparison of Simultaneous or Delayed Liver Surgery for Limited Synchronous Colorectal Metastases," British Journal of Surgery, Vol. 97, No. 8, 2010, pp. 1279-1289. doi:10.1002/bjs.7106 